\title{
Pairwise joint probability propagation in BICM-ID
}

\author{
Florence Alberge \\ Laboratoire des Signaux et Systèmes \\ Univ. Paris-Sud, UMR8506 Orsay, F-91405; CNRS, Gif-sur-Yvette, F-91192; \\ Supelec, Gif-sur-Yvette, F-91192 \\ Email:alberge@1ss.supelec.fr
}

\begin{abstract}
The iterative decoding of BICM is analyzed from an optimization point of view. The objective function underlying the standard iterative decoding is a coarse approximation of the optimal Maximum Likelihood Decoding. This approximation is suitable when large block of data are processed but may be critical for short blocks. In this contribution, it is proved that refined approximations can be obtained by propagating extrinsics on groups of bits. The particular case of pairwise joint probability propagation is investigated. Its computational complexity is similar to standard iterative decoding. Simulations show the favorable behavior of the proposed method.
\end{abstract}

\section{INTRODUCTION}

Bit-Interleaved Coded Modulation (BICM) was first suggested by Zehavi in [1] to improve the Trellis Coded Modulation performance over Rayleigh-fading channels. BICM has been used in recent standards like DVB-S2, wireless LANs, DSL and WiMax because of its flexibility and simplicity.

BICM combines error-correcting codes with higher-order modulation schemes. Although originally developed for singleinput single-output (SISO) fading channels [1][2], BICM has quickly found its way in state-of-the-art multi-antenna systems (MIMO systems). BICM can combine high data rates while still maintaining high diversity. In BICM, the diversity order is increased by using bit-interleavers instead of symbol interleavers. This improvement is achieved at the expense of a reduced minimum Euclidean distance leading to a degradation over non-fading Gaussian channels [1]. This drawback can be overcome by using iterative decoding (BICM-ID) at the receiver where the channel decoder and the demodulator exchange extrinsic information in a Turbo-like process [3]. The iterative decoding scheme used in BICM-ID is very similar to serially concatenated turbo-decoders. In BICM-ID, the inner decoder is replaced by a demapper which is less computationally demanding than a decoding step. The turbolike iterative decoding was not originally introduced as a solution to an optimization problem, rendering its analysis difficult. In [4], it is proved that iterative decoding proceeds from an approximation of the optimal Maximum Likelihood Decoding (MLD). The extrinsics propagation is a natural consequence of the Jacobi/Gauss-Seidel scheduling which is used to obtain an iterative procedure.

The literature on BICM systems is mainly focused on the design of the three constitutive elements of the transmitter namely the encoder, the interleaver and the mapping strategy. We conjecture that the algorithmic procedure used at the receiver is also a key element for improving the performance of the whole system. This paper provides a generalization of the framework in [4] taking inspiration from [5]. The scheme in [4] is general and can be applied for seeking the maximum of a sum of functions with discrete variables. The approximation is meaningful when the direct optimization is rendered intractable by forming the sum of the functions. In section II-A, a family of approximate objective functions, with key parameter $r$, is derived. A coarse approximation is obtained when $r=1$, refined approximations can be obtained by increasing $r$. Standard iterative decoding makes use of the coarse approximation $(r=1)$ which may not be enough for short blocks of data. An iterative procedure with $r=2$, is proposed in section III-B without any extra computational cost. The resulting algorithm propagates pairwise joint probabilities instead of single bit probabilities. A comparison of these iterative schemes is provided in section IV.

\section{OPTIMIZATION PROBLEM}

\section{A. General framework}

We consider the general problem of finding the maximum of a sum of functions with binary variables $x_{1}, \ldots, x_{n}$ :

$$
\mathbf{x}_{\text {opt }}=\arg \max _{\mathbf{x} \in\{0 ; 1\}^{n}} f_{\alpha}(\mathbf{x})+f_{\beta}(\mathbf{x})
$$

where $\mathbf{x}=\left(x_{1}, x_{2}, \ldots, x_{n}\right)$ and where $f_{i}(\mathbf{x}), i \in\{\alpha, \beta\}$, is a real-valued functions with $f_{i}(\mathbf{x})<+\infty$. This leads to the following optimization problem:

$$
\mathbf{x}_{\text {opt }}=\arg \max _{\mathbf{x} \in\{0 ; 1\}^{n}} p_{\alpha}(\mathbf{x}) p_{\beta}(\mathbf{x})
$$

where $p_{i}(x)=e^{f_{i}(x)}$. A convenient formulation of (2) can be obtained by replacing the discrete variable $\mathrm{x}$ with the continuous variable $\mathbf{p}(\mathbf{x})$ as:

$$
p_{\text {opt }}=\arg \max _{p \in \mathcal{E}} \sum_{\mathbf{x}} p_{\alpha}(\mathbf{x}) p_{\beta}(\mathbf{x}) p(\mathbf{x})
$$

where $\mathcal{E}$ contains all the possible PMFs on $\mathbf{x}$. A global optimum $p_{\text {opt }}(x)$ is the Kronecker delta function $p_{\text {opt }}(x)=\delta_{x, x_{\text {opt }}}$ since another weighting with the constraint $\sum_{x} p(x)=1$ produces a lower value of the objective function. As a consequence, the maximization of (3) gives a solution to the original problem in (1).

We assume that the intractability of (3) arises from forming the product $p_{\alpha}(x) p_{\beta}(x)$. Our framework of approximations will be restricted to problems where the expectations with $p_{\alpha}$ or $p_{\beta}$ and relative to some suitable $p(x)$ are tractable. A 
class of suboptimal objective functions can be obtained by relaxing the full-agreement for $p_{\alpha}(\mathbf{x})$ and $p_{\beta}(\mathbf{x})$ over $n$ bits $\left(x_{1}, x_{2}, \ldots x_{n}\right)$ to a partial agreement over a subset of $r$ bits $(r<n)$. The set $\mathcal{E}_{r}$ of $r$-factorized PMFs is defined without any loss of generality as $\mathcal{E}_{r}=\left\{q \in \mathcal{E}: q(\mathbf{x})=q_{1}\left(\widetilde{\mathbf{x}}_{1}^{r}\right) \times \ldots \times\right.$ $\left.q_{k}\left(\widetilde{\mathbf{x}}_{k}^{r}\right) \times \ldots \times q_{u}\left(\widetilde{\mathbf{x}}_{u}^{r}\right)\right\}$ with $\widetilde{\mathbf{x}}_{k}^{r}=\left(x_{(k-1) r+1}, \ldots, x_{k r}\right)$ and $u r=n$. For any value of $r$, the global optimum $p_{\text {opt }}=\delta_{x, x_{o p t}}$ is a $r$-factorized PMF. The continuous variable $p(\mathbf{x})$ is now split into two intermediate variables $l(\mathbf{x})$ and $q(\mathbf{x})$ such that: $p(\mathbf{x})=l(\mathbf{x}) q(\mathbf{x})$ and $l, q \in \mathcal{E}_{r}$. The problem in (3) reads

$$
\left(l_{\text {opt }}, q_{\text {opt }}\right)=\arg \max _{l, q \in \mathcal{E}_{r}} \sum_{\mathbf{x}} p_{\alpha}(\mathbf{x}) p_{\beta}(\mathbf{x}) l(\mathbf{x}) q(\mathbf{x})
$$

with global solution $l_{\text {opt }}(x)=q_{\text {opt }}(x)=\delta_{x, x_{o p t}}$. The formulation in (4) is equivalent to the original problem since the two solutions $l_{\text {opt }}, q_{\text {opt }}$ both select the optimal sequence $x_{o p t}$. Let $\mathcal{C}_{\text {opt }}$ denote the objective function in (4). By grouping together the terms with the same value of $\widetilde{\mathbf{x}}_{k}$, we obtain another expression for the original problem

$$
\left(l_{\text {opt }}, q_{\text {opt }}\right)=\arg \max _{l, q \in \mathcal{E}_{r}} \sum_{\widetilde{\mathbf{x}}_{k}^{r}} \sum_{\mathbf{x}: \widetilde{\mathbf{x}}_{k}^{r}} p_{\alpha}(\mathbf{x}) p_{\beta}(\mathbf{x}) l(\mathbf{x}) q(\mathbf{x})
$$

We are ready for applying the main approximation which consists in replacing the marginals of the product by the product of the marginals leading to the suboptimal problem:

$$
\widetilde{C}_{k, r}(l, q)=\sum_{\widetilde{\mathbf{x}}_{k}^{r}}\left(\sum_{\mathbf{x}: \widetilde{\mathbf{x}}_{k}^{r}} p_{\alpha}(\mathbf{x}) q(\mathbf{x}) \sum_{\mathbf{x}^{\prime}: \widetilde{\mathbf{x}}_{k}^{r}} p_{\beta}\left(\mathbf{x}^{\prime}\right) l\left(\mathbf{x}^{\prime}\right)\right)
$$

This approximation deserves some comments. From the optimal problem in (5), we have derived a family of $\frac{n}{r}$ approximate objective functions. The objective function $\widetilde{\mathcal{C}}_{k, r}(l, q)$ is the sum of all the instances of the product $p_{\alpha}(\mathbf{x}) q(\mathbf{x}) p_{\beta}\left(\mathbf{x}^{\prime}\right) l\left(\mathbf{x}^{\prime}\right)$ for pairs $\left(\mathbf{x}, \mathbf{x}^{\prime}\right)$ such that $\widetilde{\mathbf{x}}_{k}^{r}=\widetilde{\mathbf{x}}^{\prime}{ }_{k}$ (ie agreement for the $r$ bits of $\widetilde{\mathbf{x}}_{k}^{r}$ and $\widetilde{\mathbf{x}}^{\prime}{ }_{k}^{r}$ ). This suggests that $\widetilde{\mathcal{C}}_{k, r}(l, q)$ should be used for determining the optimal value of the $r$ common bits. For that purpose, $l$ and $q$ are restricted to $\mathcal{E}_{r}$. The original problem is thus replaced by the next $\frac{n}{r}$ optimization problems:

$$
\arg \max _{l_{k}, q_{k} \in \mathcal{E}} \widetilde{\mathcal{C}}_{k, r}(l, q) \quad 1 \leq k \leq \frac{n}{r}
$$

This is a distributive optimization strategy. The joint objective function is defined as $\widetilde{\mathcal{C}}_{r}(l, q)=\sum_{k=1}^{\frac{n}{r}} \widetilde{\mathcal{C}}_{k, r}(l, q)$. The value of $\widetilde{\mathcal{C}_{r}}(l, q)$ is a performance rating on the efficiency of the joint optimization process. Using synchronization considerations, we can also observe that terms such that $\tilde{\mathbf{x}}_{k}^{r}=\tilde{\mathbf{x}}_{k}^{\prime} \forall k$ will appear on every objective function $\widetilde{\mathcal{C}}_{k, r}$ whereas terms such that $\tilde{\mathbf{x}}_{k}^{r} \neq{\tilde{\mathbf{x}^{\prime}}}_{k}^{r} \forall k$ will never appear. With this line of arguments, we obtain a new expression for the joint objective function

$$
\begin{aligned}
\widetilde{\mathcal{C}}_{r}(l, q) & =\frac{n}{r} \sum_{v=0}^{\frac{n}{r}}\left(1-v \frac{r}{n}\right) \mathcal{N}_{v}(l, q) \\
& =\frac{n}{r}\left(\mathcal{C}_{\text {opt }}+\sum_{v=1}^{\frac{n}{r}-1}\left(1-\frac{r}{n} v\right) \mathcal{N}_{v}(l, q)\right)
\end{aligned}
$$

where $\mathcal{N}_{v}(l, q)$ contains the products $p_{\alpha}(\mathbf{x}) q(\mathbf{x}) \underset{\sim}{p_{\beta}}\left(\mathbf{x}^{\prime}\right) l\left(\mathbf{x}^{\prime}\right)$ with $v$ elements in common $\left(\tilde{\mathbf{x}}_{i}^{r}, \tilde{\mathbf{x}}^{\prime}{ }_{i}^{r}\right)\left(\tilde{\mathbf{x}}_{i}^{r} \neq \tilde{\mathbf{x}}_{i}^{\prime}{ }_{i}, i \in \mathcal{I}\right.$, $\operatorname{card}(\mathcal{I})=v$ ) and $n / r-v$ distinct elements $\left(\tilde{\mathbf{x}}_{j}^{r}, \tilde{\mathbf{x}}^{r}{ }_{j}\right)$ $\left(\tilde{\mathbf{x}}_{j}^{r}=\tilde{\mathbf{x}}^{\prime}{ }_{j}^{r}, j \in \mathcal{J}, \operatorname{card}(\mathcal{J})=\frac{n}{r}-v\right)$. It turns out that $\widetilde{\mathcal{C}}_{r}(l, q)$ is a weighted sum involving the original criterion $\mathcal{C}_{\text {opt }}$ and extra terms due to the approximation. The choice of $r$ is a trade-off between fidelity to the original criterion $(r=n)$ and computational complexity $(r=1)$. It is interesting to note that this approximation shares common ideas with [6], [7] developed in the context of belief propagation.

\section{B. Iterative maximization}

The $k^{t h}$ approximate optimization problem (7) reads:

$$
\arg \max _{l_{k}, q_{k} \in \mathcal{E}_{r}} \sum_{\widetilde{\mathbf{x}}_{k}^{r}} q_{k}\left(\widetilde{\mathbf{x}}_{k}^{r}\right) l_{k}\left(\widetilde{\mathbf{x}}_{k}^{r}\right) f_{\widetilde{\mathbf{x}}_{k}^{r}}\left(p_{\alpha}, q_{-k}\right) f_{\widetilde{\mathbf{x}}_{k}^{r}}\left(p_{\beta}, l_{-k}\right)
$$

with $f_{\widetilde{\mathbf{x}}_{k}^{r}}\left(p_{\alpha}, q_{-k}\right)=\sum_{\mathbf{x}: \widetilde{\mathbf{x}}_{k}^{r}} p_{\alpha}(\mathbf{x}) \prod_{i \neq k} q_{i}\left(\widetilde{\mathbf{x}}_{i}^{r}\right)$ and where $q_{-k}(\mathbf{x})=\prod_{i \neq k} q_{i}\left(\widetilde{\mathbf{x}}_{i}^{r}\right)$. The same definitions hold for $f_{\widetilde{\mathbf{x}}_{k}^{r}}\left(p_{\beta}, l_{-k}\right)$ and $l_{-k}$. Since $0 \leq q_{k}\left(\widetilde{\mathbf{x}}_{k}^{r}\right) l_{k}\left(\widetilde{\mathbf{x}}_{k}^{r}\right) \leq 1$, a global optimum is given by $q_{k, \text { opt }}\left(\widetilde{\mathbf{x}}_{k}^{r}\right) l_{k, \text { opt }}\left(\widetilde{\mathbf{x}}_{k}^{r}\right)=\delta_{\widetilde{\mathbf{x}}_{k}^{r}, \widehat{\mathbf{x}}_{k}^{r}}$ where $\widehat{\mathbf{x}}_{k, o p t}^{r}$ is a global maximum (with respect to $\widetilde{\mathbf{x}}_{k}^{r}$ ) of $f_{\widetilde{\mathbf{x}}_{k}^{r}}\left(p_{\alpha}, q_{-k}\right) f_{\widetilde{\mathbf{x}}_{k}^{r}}\left(p_{\beta}, l_{-k}\right)$. This is an hard-decision process. In order to limit the number of local maxima in the iterative scheme, soft-decisions are preferred [8]:

$$
q_{k}\left(\widetilde{\mathbf{x}}_{k}^{r}\right) l_{k}\left(\widetilde{\mathbf{x}}_{k}^{r}\right) \propto f_{\widetilde{\mathbf{x}}_{k}^{r}}\left(p_{\alpha}, q_{-k}\right) f_{\widetilde{\mathbf{x}}_{k}^{r}}\left(p_{\beta}, l_{-k}\right)
$$

The choice of a scheduling gives the algorithm. Without prior information, a natural choice for $l^{(0)}$ and $q^{(0)}$ is uniform distributions. An hybrid Jacobi/Gauss-Seidel scheduling can be applied:

Step 1: Computation of $q_{k}^{(i t)}$ for $1 \leq k \leq \frac{n}{r}$ based on the estimates of the previous iteration $l_{-k}^{(i t-1)}, q_{-k}^{(i t-1)}$

Step 2: Computation of $l_{k}^{(i t)}$ for $1 \leq k \leq \frac{n}{r}$ based on available estimates $l_{-k}^{(i t-1)}, q_{-k}^{(i t)}$

Stop if $l_{k}^{(i t)} q_{k}^{(i t)}=l_{k}^{(i t-1)} q_{k}^{(i t)}$ for $1 \leq k \leq \frac{n}{r}$

The updates are:

$$
\begin{array}{r}
q_{k}^{(i t)}\left(\widetilde{\mathbf{x}}_{k}^{r}\right) \propto f_{\widetilde{\mathbf{x}}_{k}^{r}}\left(p_{\alpha}, q_{-k}^{(0)}\right) f_{\widetilde{\mathbf{x}}_{k}^{r}}\left(p_{\beta}, l_{-k}^{(i t-1)}\right) \\
l_{k}^{(i t)}\left(\widetilde{\mathbf{x}}_{k}^{r}\right) \propto \frac{f_{\widetilde{\mathbf{x}}_{k}^{r}}\left(p_{\alpha}, q_{-k}^{(i t)}\right)}{f_{\widetilde{\mathbf{x}}_{k}^{r}}\left(p_{\alpha}, q_{-k}^{(0)}\right)}
\end{array}
$$

The number of quantities to be computed at each iteration (1213) is $\frac{n}{r} 2^{r+1}$. The value of $\frac{n}{r} 2^{r+1}$ is linearly increasing with $n$ and exponentially increasing with $r$. With $r=2$ and also with $r=1, \frac{n}{r} 2^{r+1}=4 n$. In the next section, this general framework is applied to BICM.

\section{ApplicAtion to BICM}

\section{A. Transmission scheme and iterative decoding}

A BICM system [2] is built from a serial concatenation of a convolutional encoder, a bit interleaver and an $M$-ary bits-to-symbol mapping (where $M=2^{m}$ ) as shown in Fig. 1. The sequence of information bits $\mathbf{b}$ of length $n_{b}$ is first encoded by a convolutional encoder to produce the output 


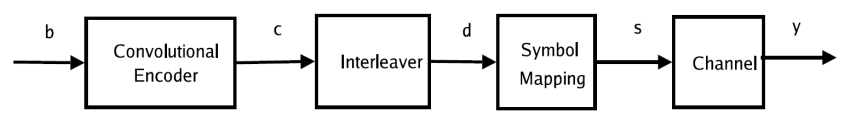

Fig. 1. BICM transmission scheme

encoded bit sequence $\mathbf{c}$ of length $n$ which is then scrambled by a bit interleaver operating on bit indexes. Let $\mathbf{d}=\pi(\mathbf{c})$ denote the interleaved sequence. Then, $m$ consecutive bits of $\mathbf{d}$ are grouped as a symbol. The transmitted signal $s_{k}$, $1 \leq k \leq n / m$, is then chosen from an $M$-ary constellation $\psi$ where $\psi$ denotes the mapping scheme. The symbols $s_{k}$ are passed over a noisy memoryless channel to get the channel outputs $y_{k}$. The maximum likelihood sequence detection reads:

$$
\widehat{\mathbf{b}}_{M L D}=\arg \max _{\mathbf{b} \in\{0,1\}^{n_{b}}} \mathbf{p}(\mathbf{y} \mid \mathbf{b})
$$

where $\mathbf{p}(\mathbf{y} \mid \mathbf{b})$ is the likelihood function which results from concatenating the encoder with the channel. Since there is a one-to-one correspondence between the binary message $\mathbf{b}$ and the interleaved sequence $\mathbf{d}$, eq. (14) is equivalent to searching $\widehat{\mathbf{d}}_{M L D}$ as:

$$
\widehat{\mathbf{d}}_{M L D}=\arg \max _{\mathbf{d} \in\{0,1\}^{n}} \mathbf{p}_{c h}(\mathbf{y} \mid \mathbf{d}) I_{c o}(\mathbf{d})
$$

where $\mathbf{p}_{c h}(\mathbf{y} \mid \mathbf{d})$ is the probability of receiving $\mathbf{y}$ when the sequence transmitted through the channel is the mapping of $\mathbf{d}$ and where $\mathbf{I}_{c o}(\mathbf{d})$ is the indicator function of the code meaning that $\mathbf{I}_{c o}(\mathbf{d})=1$ if $\mathbf{c}=\pi^{-1}(\mathbf{d})$ is a codeword and 0 elsewhere. Another way to tackle this problem consists in finding the prior PMF on $\mathbf{d}$ which maximizes the a posteriori probability of having received $\mathbf{y}$

$$
\widehat{\mathbf{p}}_{M L D}(\mathbf{d})=\arg \max _{p \in \mathcal{E}} \sum_{\mathbf{d}} \mathbf{I}_{c o}(\mathbf{d}) \mathbf{p}_{c h}(\mathbf{y} \mid \mathbf{d}) \mathbf{p}(\mathbf{d})
$$

This is exactly the optimization problem in (3). It is proved in [4] that the turbo-like iterative decoding of BICM is exactly equations (12-13) with $r=1$. Agreement is required on a unique bit $\left(\widetilde{\mathbf{x}}_{k}^{1}=d_{k}\right)$ and the iterative decoding propagates probabilities on a single bit. The involved quantities are $f_{d_{k}}\left(\mathbf{p}_{c h}(\mathbf{y} \mid \mathbf{d}), l_{-k}^{(i t-1)}\right)$ and $f_{d_{k}}\left(\mathbf{I}_{c o}(\mathbf{d}), q_{-k}^{(i t)}\right)$. These quantities are respectively the output given by the demapping sub-block and the second one is the output given by a BCJR algorithm [9] at the decoder side. In the coding community, $l_{k}\left(d_{k}\right), q_{k}\left(d_{k}\right)$ are usually called extrinsics and $l_{k}\left(d_{k}\right) q_{k}\left(d_{k}\right)$ is the APP (A Posteriori Probability). We have seen in section II-A that $\widetilde{\mathcal{C}}_{r} \stackrel{r \rightarrow n}{\longrightarrow} \mathcal{C}_{\text {opt }}$. In BICM-ID, $r$ is set to 1 leading to a rough approximation of the MLD whereas higher values of $r$ are expected to provide an accurate approximation. Complexity is a critical issue in BICM and in turbo-like algorithms in general. We mentioned in the previous section that the number of exchanged quantities is the same for both $r=1$ and $r=2$ whereas this number is increasing exponentially with $r$ when $r>2$. In the next section, a new algorithm based on (12-13) is proposed for BICM with $r=2$.

\section{B. Extended algorithm}

Considering $r=2, \widetilde{\mathbf{x}}_{k}^{2}$ is a slice of two bits that can be either consecutive within the interleaved sequence $\mathbf{d}$, consecutive within the coded sequence $\mathbf{c}$ or chosen with another one-toone mapping. The updates in (12-13) reads

$$
\begin{array}{r}
q_{k}^{(i t)}\left(\widetilde{\mathbf{x}}_{k}^{2}\right) \propto \sum_{\mathbf{x}: \widetilde{\mathbf{x}}_{k}^{2}} \mathbf{p}_{c h}(\mathbf{y} \mid \mathbf{d}) \prod_{i \neq k} l_{i}^{(i t-1)}\left(\widetilde{\mathbf{x}}_{i}^{2}\right) \\
l_{k}^{(i t)}\left(\widetilde{\mathbf{x}}_{k}^{2}\right) \propto \sum_{\mathbf{x}: \widetilde{\mathbf{x}}_{k}^{2}} \mathbf{I}_{c o}(\mathbf{d}) \prod_{i \neq k} q_{i}^{(i t)}\left(\widetilde{\mathbf{x}}_{i}^{2}\right)
\end{array}
$$

Since, when $\mathbf{q}^{(0)}$ is a uniform distribution, $f_{\widetilde{\mathbf{x}}_{k}^{2}}\left(\mathbf{I}_{c o}(\mathbf{d}), q_{-k}^{(0)}\right)=$ $\frac{1}{4} \forall \widetilde{\mathbf{x}}_{k}^{2} \in\{0,1\}^{2}$. For a memoryless channel, $\mathbf{p}_{c h}(\mathbf{y}$ d) $=\prod_{j=1} \mathbf{p}_{c h}\left(y_{j} \mid s_{j}\right)$ with $s_{j}=\psi\left(d_{m(j-1)+1}, \ldots, d_{j m}\right)$. The demapping task is less complex if the two bits $\left(d_{\Omega(2(k-1)+1)}, d_{\Omega(2 k)}\right)$ of $\widetilde{\mathbf{x}}_{k}^{2}$ belong to the same symbol,

$$
\widetilde{\mathbf{x}}_{k}^{2}=\left(d_{2(k-1)+1}, d_{2 k}\right)
$$

In standard BICM systems $(r=1)$ with convolutive code, the decoding task may be performed by a BCJR algorithm [9]. The BCJR algorithm returns single variable probability. An extension of the BCJR to compute higher order joint probability has been published in [10]. As a special case, this algorithm computes pairwise joint probabilities. The structure of a BCJR algorithm is based on the trellis representation of a convolutive code. The probability of transition between two states is connected with the probability of the source. In the iterative decoding context of BICM, the probability of the source is the extrinsic $q$ which is used as a prior information. In a trellis representation, the transitions between two states are associated to a particular value of a group of consecutive bits in the sequence $\mathbf{c}$ (a group of 2 bits if a convolutive code with rate $R=1 / 2$ is considered). Thus consecutive bits of the coded sequence $\mathbf{c}$ should be assigned to $\widetilde{\mathbf{x}}_{k}^{2}$,

$$
\left.\widetilde{\mathbf{x}}_{k}^{2}=\left(c_{2(k-1)+1}, c_{2 k}\right)=\left(d_{\pi(2(k-1)+1)}, d_{\pi(2 k)}\right)\right)
$$

BICM makes uses of a bit-interleaving. In this case, (19) and (20) are antagonists requirements that can not be met at the same time. The propagation of pairwise probabilities is possible by changing the standard iterative decoding. Three modified schemes are possible:

Relax requirement (19) (demapping): the demapping is more complex since the whole sequence has to be considered for the marginal computation in (17).

Relax requirement (20) (decoding): the decoding task can not be handled by a BCJR algorithm leading to an extra computational cost.

Maintain both requirements (interleaver): in this case, the bitinterleaver is replaced by a pairwise interleaver ie both bits $c_{2(k-1)+1}$ and $c_{2 k}$ belong to the same pair, the interleaver operates on index $k$.

The last solution maintains the computational cost to the level of the standard iterative decoding. The others strategies are not under the scope of this paper. The role of the interleaver in fading channels is to guarantee that consecutive coded 
bits are affected by independent fades. In both fading and non-fading channels, the interleaver breaks the dependency between consecutive elements of the sequence $d$. This is fundamental to obtain efficient approximations of (5) even with small value of $r$. The restriction on the interleaver may lead to a degraded channel probability with fading channels but not necessarily with the AWGN channel. However an interleaver compatible with the value of $r$ is needed for an accurate approximation. In the simulation part, this new strategy is compared to the usual one for the AWGN channel. Preliminary results are also reported for channels with fading.

\section{Simulations}

A BICM transmitter is considered with $(115,177)_{8}$ convolutive code of rate $\frac{1}{2}$ and a 16-QAM constellation. Several associations mapping/interleaver/synchronization are compared (see table I). It is proved in [11] that the performance of BICM can be improved in non-fading channels when the interleaver takes a trivial form meaning that the bits are not interleaved at all provided that an appropriate mapping is chosen. The mapping under consideration in [11] is the binary reflected Gray code (BRGC) of [12]. Under this setting, the modified scheme is expected to provide some improvements over the usual iterative decoding $(r=1)$. In the simulations, the number of iterations is 10 . When using BRGC however the iterative decoding does not take benefits of the iterations since the BER does not decrease after iteration 2. In this paper, short blocks are considered. The number of information bits is $n_{b}=400$ and the number of encoded/interleaved bits is $n=800$. It is proved in [8] that the value of the joint

\begin{tabular}{|l||l|l|l|}
\hline Name & Mapping & Interleaver & $r$ \\
\hline Gray & BRGC & trivial & 1 \\
\hline SP-1bit & Set Partitioning & bit-interleaver & 1 \\
\hline SP-2bit & Set Partitioning & pairwise-interleaver & 2 \\
\hline
\end{tabular}

TABLE I

PLOT LABELING

objective function is a performance rating on the efficiency of the optimization process and is also indicative of the vicinity of the solution to the maximum likelihood optimal. The value of $\frac{r}{n} \widetilde{\mathcal{C}_{r}}(l, q)$ is plotted as a function of the number of errors (on the encoded bits) in the particular case of an AWGN channel and $\frac{E_{b}}{N_{0}}=4.5 \mathrm{~dB}$. The results are reported in Fig. 2 for Gray and in Fig 3 for SP-1bit and SP-2bit. We observe the following. With Gray, all points are located in the same area. There is no local maxima but a significant number of frames exhibits more than 10 errors. With SP-1bit, a threshold $T_{1}$ can be applied to separate MLD solutions from local maxima. We will choose in the following $T_{1}=0.6$. When $\frac{r}{n} \widetilde{\mathcal{C}}_{r}(l, q)$ is above $T_{1}$, the average number of errors on the encoded bits is 0.0633 (smaller than with Gray) but for an important number of frames the algorithm fails to converge to the MLD. With SP-2bit, the threshold is fixed to $T_{2}=0.95$ for SP-2bit. When $\frac{r}{n} \widetilde{\mathcal{C}}_{r}(l, q)$ is above $T_{2}$, the average number of errors on the encoded bits is 0.0225 . We also observe frames in which

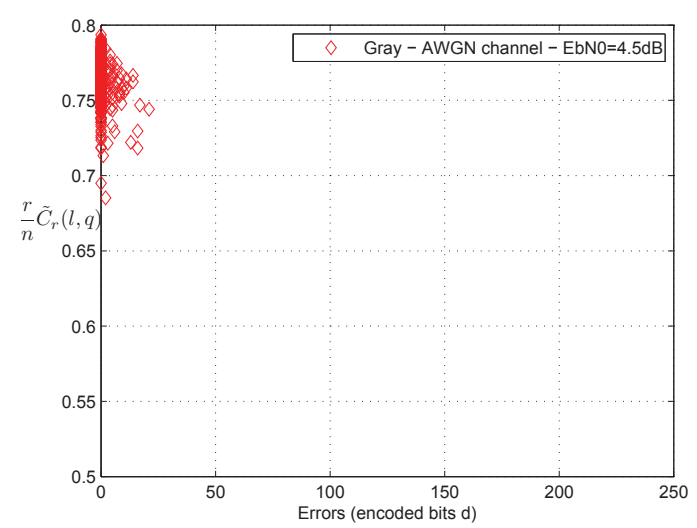

Fig. 2. Plot of pairs (errors, $\widetilde{\mathcal{C}}_{r}(l, q)$ ), Gray settings, AWGN channel, $\frac{E_{b}}{N_{0}}=4.5 d B$

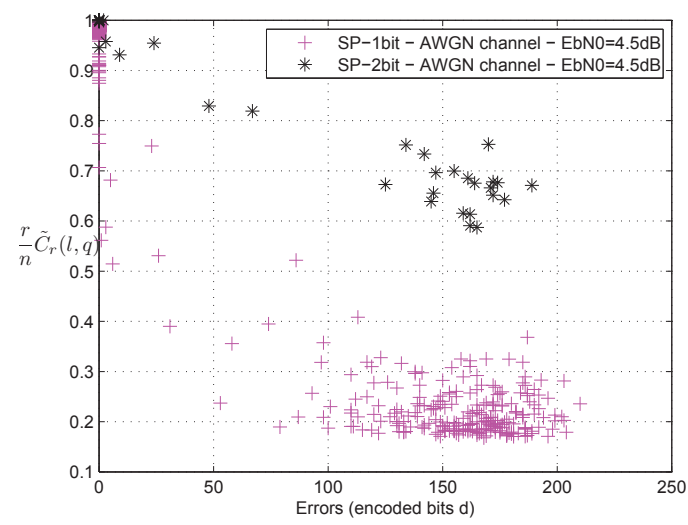

Fig. 3. Plot of pairs (errors, $\widetilde{\mathcal{C}}_{r}(l, q)$ ) for SP-1bit and SP-2bit settings, AWGN channel, $\frac{E_{b}}{N_{0}}=4.5 \mathrm{~dB}$

the algorithm fails to converge to the MLD. The number of such pathological frames is 275 in SP-1bit and 26 in SP-2bit (among a total of 1000 frames). This is a first illustration of the superior properties of SP-2bit over SP-1bit. The Bit Error Rate (BER) is plotted as a function of $\frac{E_{b}}{N_{0}}$ for the AWGN channel in Fig. 4. The experiment is conducted with the settings in table I. The BER for curves with label Gray, SP-1bit and SP-2bit was obtained by computation over all the frames. In the two other curves, a threshold is applied and the BER is computed only for frames such that $\frac{r}{n} \widetilde{\mathcal{C}}_{r}(l, q)>T_{r}$ (at iteration 10). The proportion of frames above $T_{r}(r=1, r=2)$ is given in Fig. 5. This is indicative of the proportion of frames with MLD solution at the end of the iterative process. We conjectured that choosing $r=2$ rather than $r=1$ could decrease the number of local maxima. This is confirmed by the simulation. For example, with $\frac{E_{b}}{N_{0}}=4 \mathrm{~dB}$, the proportion of frames with MLD solution at iteration 10 is $88 \%$ with $r=2$ and $38.73 \%$ with $r=1$ and the BER computed among those frames is slightly better with $r=2$ than with $r=1$. The global BER 


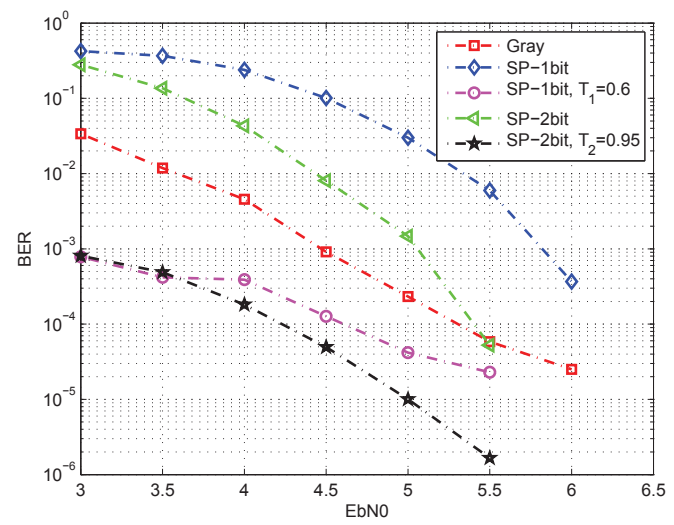

Fig. 4. BER versus $E b / N_{0}$ over AWGN channel

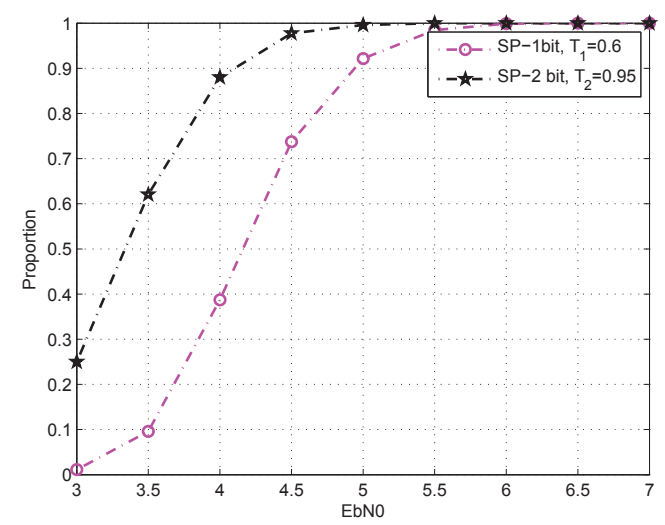

Fig. 5. Proportion of frames above threshold vs $E b / N_{0}$

(among the whole frames) is improved with $r=2$ (compared to $r=1$ ) due to the reduction of converging sequences toward a local maximum. The good performance obtained with BRGC mapping is due to the total absence of such sequences. In SP1 bit and SP-2bit, it is possible to detect, at the receiver, MLD solution based on the value reached by the objective function. For an application where frames can be emitted again or with cyclic redundancy check (CRC), SP-2bits is an interesting alternative. For example, with $\frac{E_{b}}{N_{0}}=5.5 d B$, the BER (over the whole frames) is $5.510^{-5}$ for Gray and SP-2bit. In SP2bit, the pathological sequences can be identified (1.67 10 of the total). Among the remaining sequences (99.98\%), the BER is $1.6710^{-6}$. The same kind of experiment is conducted with the fading channel. The BER is plotted in Fig. 6, the proportions of frames above treshold are not presented here due to lack of space. However, the curves obtained are very similar to Fig. 5. SP-2bit outperforms SP-1bit thanks to a drastic reduction of the number of pathological frames. For $E b N 0>7.5 d B$, the bit-interleaver gives an advantage to SP1bit. An hybrid structure (pairwise interleaver / bit interleaver) is currently under study. Further results will be reported.

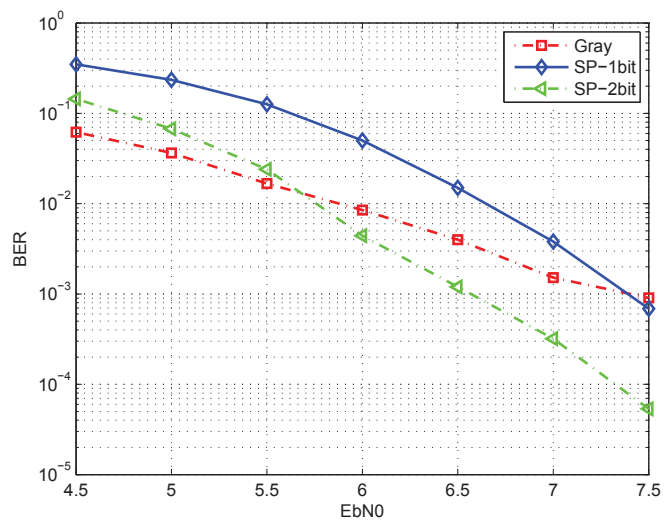

Fig. 6. BER versus $E b / N_{0}$ over fading channel

\section{CONCLUSION}

In this paper, the iterative decoding of BICM is considered from an optimization point of view. New algorithmic schemes are specifically developed to improve the performance when short blocks of data are processed. In particular, we propose to propagate pairwise joint probability instead of probability on single bits. The number of local maxima is significantly reduced. A threshold on the objective function can be used for selecting frames with MLD solutions. The BER is improved for both AWGN and fading channel. The computational cost of the proposed method is similar to standard iterative decoding.

\section{REFERENCES}

[1] E. Zehavi, "8-PSK trellis codes for a Rayleigh fading channel," IEEE Trans. on Commun., vol. 40, pp. 873-883, May 1992.

[2] G. Caire, G.Taricco, and E. Biglieri, "Bit-interleaved coded modulation," IEEE Trans. on Inform. Theory, vol. 4, pp. 927-946, May 1998.

[3] X. Li, A. Chindapol, and J.A. Ritcey, "Bit interleaved coded modulation with iterative decoding and 8-PSK signaling," IEEE trans Commun., vol. 50, pp. 1250-1257, Aug 2002.

[4] F. Alberge, Z. Naja, and P. Duhamel, "From Maximum Likelihood to Iterative Decoding," in ICASSP Proc., Prague, Czech Republic, 22-27 May 2011

[5] J. M. Walsh, P.A. Regalia, and C. R. Johnson, "Turbo decoding as Iterative Constrained Maximum-Likelihood Sequence Detection," IEEE Trans. on Inform. Theory, vol. 52, pp. 5426-5437, Dec. 2006.

[6] P. Pakzad and V. Anantharaman, "Estimation and marginalization using Kikushi approximation methods," Neural Comput., vol. 17, no. 8, pp. 1836-1873, 2005.

[7] J. Yedidia, W. Freeman, and Y. Weiss, "Constructing free-energy approximations and generalized belief propagation algorithms," IEEE Transactions on Information Theory, , no. 7, pp. 2282-2312, Jul. 2005

[8] F. Alberge, "A game-theoretic interpretation of iterative decoding," in EUSIPCO, Barcelona, Spain, 29 Aug. - 2 Sept. 2011, vol. 1, pp. 76-80.

[9] L.R. Bahl, J. Cocke, F. Jelinek, and J.Raviv, "Optimal Decoding of Linear Codes for Minimizing Symbol Error Rate," IEEE Trans. on Inform. Theory, pp. 284-287, March 1974.

[10] J.H. Gunther, D. Keller, and T.K. Moon, "A generalized BCJR algorithm and its use in turbo synchronization," in ICASSP, Philadelphia, PA, USA, 18-23 May 2005, vol. 3, pp. 837-840.

[11] A. Alvarado, L. Szczecinski, and E. Agrell, "On BICM receivers for TCM transmission," IEEE Transactions on Communications, vol. 59, no. 10, pp. 2692-2702, 2011.

[12] E. Agrell, J. Lassing, E.G. Strø̈m, and T. Ottosson, "On the optimality of the binary reflected Gray code," IEEE Trans. Inf. Theory, vol. 50, no. 12, pp. 3170-3182, 2004. 